Background The maintenance of physical and cognitive function for the maximal period of time is a key component of healthy ageing, associated with continued independent living, better quality of life and reduced morbidity and mortality risk. There is growing interest in investigating life course influences on healthy ageing to identify potential intervention targets, beyond those already identified by chronic disease epidemiology.

Using data from the MRC National Survey of Health and Development (NSHD), we assess:

1. the relative importance of socioeconomic position (SEP) in childhood and adulthood across a range of physical and cognitive functional ageing outcomes at age 60-64; 2 . the contribution of key social, behavioural and developmental intermediary factors to the relationship between child SEP and functional ageing

Methods Ageing outcomes (lung function, grip strength, chair rise time, standing balance, timed up and go (TUG), verbal memory, processing speed and simple reaction time) were regressed on childhood and adult SEP ridit scores (calculated as the proportion of the population higher than the midpoint for each category) and sex. The ridit score coefficient is the slope index of inequality (SII), interpreted as the absolute difference in outcome between the hypothetical top and bottom of the SEP gradient. These were converted to the relative index of inequality to provide an estimate of the relative SEP difference across outcomes and over time. Regression models were then adjusted to examine the influence of potential mediators (education, smoking, BMI, height and childhood cognition) on the SII.

Results Substantial childhood and adult socioeconomic gradients were observed in all physical and cognitive outcomes. The hypothetical top of the childhood SEP distribution performed between 9 and 18 per cent better, relative to the hypothetical bottom ( $p \leq$ 0.05 for association between childhood SEP with all outcomes). These associations persisted on adjustment for adult SEP, with the exception of standing balance. The hypothetical top of the adult SEP distribution performed between 6 and 26 per cent better, relative to the hypothetical bottom ( $p \leq 0.05$ for association between adult SEP with all outcomes). With the exception of processing speed (in women only), chair rise time, and TUG, associations between adult SEP and outcomes persisted on adjustment for childhood SEP. Adjusting for potential mediators attenuated specific associations

Conclusion Child and adult SEP were independently associated with physical and cognitive ageing at age 60-64. The different social, behavioural and developmental pathways partly mediating these associations may guide appropriate intervention strategies.

\section{PS20 PROSPECTIVE AUDIT OF PAEDIATRIC UNINTENTIONAL INJURY}

doi:10.1136/jech-2012-201753.119

V Jessop. Centre for Primary Care and Public Health, Queen Mary University of London, London, UK

Background Injury is a leading cause of death among children and adolescents and around $16 \%$ of the world's burden of disease can be attributed to injury, reflecting the disproportionate burden of injuries among young people and added years of life lived with disability. The majority of injuries can be prevented or at least controlled and the costs of prevention are much lower than the costs of the consequences of injuries.

There is a crucial lack of data and injury surveillance systems to inform prevention. In high income countries it has been shown that many child and adolescent injuries can be prevented through careful analysis and appropriate action. In January 2012 The Department of Health published 'A public health outcomes framework for England, 2013-2016', which includes 'Hospital admissions caused by unintentional and deliberate injuries in under $18 s^{\prime}$ as an indicator of population health.
Research has shown that a relationship exists between increasing numbers of children presenting to Accident and Emergency with injuries and higher levels of area level socioeconomic deprivation. The Indices of Deprivation 2010 show that the London Borough of Tower Hamlets (LBTH) remains one of the most deprived areas in the country.

Methods A prospective audit has been designed for use in The Paediatric Emergency Department at The Royal London Hospital in the LBTH. The audit tool will include the WHO core minimum dataset for injury surveillance and ICD-10 for injuries, as part of an enhanced injury dataset that has been incorporated in the College of Emergency Medicine's proposed 'emergency medicine minimum dataset'. Subsequent mapping of injuries to LBTH postcodes will allow identification of injury 'hot spots' requiring further investigation and targeted interventions.

Results A preliminary retrospective audit of paediatric unintentional injury using data collected from computerised Accident and Emergency records for children aged 0 to $<18$ years who attended Royal London Hospital between July to September 2011 showed that unintentional injury results in high rates of attendance, with $40 \%$ of children attending as consequence of unintentional injury ( $n=3,013$ attendances). The main reasons for attendance were for fractures, joint and head injuries and soft tissue inflammation. However, details of where injuries occurred, mechanisms and severity were lacking; prompting a prospective audit.

Conclusion Knowledge of the epidemiology of paediatric injury is lacking. It is imperative that routine monitoring and surveillance of paediatric injuries occurs nationally in order to inform effective injury prevention strategies.

PS21 EVALUATION OF MATERNITY CARE INTERVENTION IN
RURAL NEPAL: CAN A HEALTH PROMOTION EXERCISE
IMPROVE MATERNAL HEALTH AND SERVICE UPTAKE IN
RURAL NEPAL?

doi:10.1136/jech-2012-201753.120

${ }^{1} \mathrm{~S}$ Sharma, ${ }^{2} \mathrm{E}$ Sicuri, ${ }^{3} \mathrm{~J}$ Belizan, ${ }^{1,4}{ }^{4} \mathrm{E}$ van Teijlingen, ${ }^{4,5} \mathrm{P}$ Simkhada, ${ }^{6} \mathrm{~J}$ Stephens. ${ }^{1}$ School of Health and Social Care, Bournemouth University, Bournemouth, UK; ${ }^{2}$ Centre de Recerca en Salut Internacional de Barcelona (CRESIB), Hospital Clínic, Barcelona, Spain IInstitute of Clinical Effectiveness and Health Policy, IECS, Buenos Aires, Argentina; ${ }^{4}$ Manmohan Memorial Institute of Health Sciences, Purbanchal University, Kathmandu, Nepal; ${ }^{5}$ ScHARR, University of Sheffield, Sheffield, UK; ${ }^{6}$ Green Tara Trust, London, UK

Background In developing countries, where the majority of the world's maternal deaths occur it is recognised that to target maternal mortality within limited resources, safe motherhood strategies need to be targeted to rural areas and to the poor in order to increase access to antenatal care and delivery care. In these populations, a lack of understanding of local beliefs and practices, and the reasons for them, can hinder the development of appropriate interventions.

The Green Tara Nepal intervention, Pharping, Nepal, aims to improve the uptake of maternal care practices in rural Nepal via health promotion activities in the community. The expectation is that the measured aspect of health-seeking behaviour should improve in the intervention area relative to the control.

Methods In 2008, Green Tara Nepal (GTN), a Nepalese Non-Governmental Organisation implemented a 5 year health promotion intervention to improve maternal and neonatal health in 2 rural village development communities (VDC) in Pharping, Nepal. The GTN programme works with midwives and community health workers to target fertile women, in health promotion groups and on a one-to-one basis. During this interaction, women receive advice on health behaviours and care-seeking practice.

Two surveys were conducted a baseline (2008) and a midline (2010) on the intervention communities and in 2 control communities.; 833 women of childbearing age with their last child of less 
than 2 years old, were interviewed in this controlled before and after, cross-sectional study.

Results After the intervention the proportion of women, during their last pregnancy, attending antenatal care, at least once, increased by from $84.6 \%$ to $96.8 \%(p=0.00)$. The proportion of women who had their first prenatal visit in the first trimester was increased from $39.9 \%$ to $58.1 \%(p=0.00)$. Women attending more than 4 visits increased from $37.1 \%$ to $42.6 \%(p=0.28)$.

Conclusion This ongoing community intervention providing health promotion shows an improvement on women's attendance of antenatal care.

\section{PS22 LIFE COURSE SOCIO-ECONOMIC POSITION AND QUALITY OF LIFE IN ADULTHOOD: A SYSTEMATIC REVIEW OF LIFE COURSE MODELS}

doi:10.1136/jech-2012-201753.121

${ }^{1} \mathrm{C}$ Niedzwiedz, ${ }^{2}$ SV Katikireddi, 'J Pell, 'R Mitchell. 'Institute of Health \& Wellbeing, University of Glasgow, Glasgow, UK; ${ }^{2}$ Evaluating the Health Effects of Social Interventions Programme, MRC/CSO Social \& Public Health Sciences Unit, Glasgow, UK

Background Measurement and determinants of quality of life are of increasing interest to researchers and policymakers. A relationship between current socio-economic position and subjective quality of life has been demonstrated, using wellbeing, life and needs satisfaction approaches. Less is known regarding the influence of different socio-economic trajectories across the life course and their subsequent effect on quality of life. Several conceptual models have been proposed to help explain potential life course effects on health, including accumulation, latent, pathway and social mobility models. This systematic review aimed to apply these models to studies investigating life course socio-economic effects on quality of life, in order to assess which model(s) best described any relationship.

Methods A review protocol was developed detailing explicit inclusion and exclusion criteria, search terms, data extraction items and quality appraisal procedures. Literature searches were performed in 12 electronic databases during January 2012 and the references and citations of included articles were checked for additional relevant articles. Narrative synthesis was used to analyse the results and studies were categorised into groups based on the life course design implemented.

Results After screening 7,566 records, 12 studies met the eligibility criteria. The included articles used data from 10 different datasets and five countries. Study quality varied and heterogeneity was high. Five studies assessed the latent model, two assessed the pathway model and three tested the accumulation model. Seven studies assessed social mobility models (inter-generational mobility in three, intra-generational mobility in five and one included both types). More evidence was found to support the latent model among women (but results were contradictory) and mixed evidence was found for intra-generational mobility and pathway effects. Associations also tended to vary by gender. Few studies were identified for accumulation and inter-generational mobility effects and heterogeneity of these studies resulted in limited synthesis.

Conclusion A lack of available and comparable evidence prevented the identification of the optimal life course model(s). Different target populations, outcomes and methodologies used between studies likely contributed to the mix of results. To improve the potential for synthesis in this area, future studies should aim to increase study comparability. Recommendations include testing all life course models within studies and the use of multiple measures of socioeconomic position and quality of life. To enable investigation of between-country differences, the increased collection, harmonisation and utilisation of comparable cross-national data would be beneficial.

\section{PS23 HOW WELL DOES THE NHS SUPPORT EMPLOYEES WHO WISH TO BREASTFEED ON RETURN TO WORK?}

doi:10.1136/jech-2012-201753.122

${ }^{1} \mathrm{LI}$ Watson, ${ }^{2} \mathrm{~J}$ Neufeind, ${ }^{2} \mathrm{E}$ George, ${ }^{3}$ Fenske. ${ }^{1}$ Department of Public Health, NHS Fife, Leven, UK; ${ }^{2}$ Playfield Institute, NHS Fife, Cupar, UK; ${ }^{3}$ Victoria Hospital, NHS Fife, Kirkcaldy, UK

Background In line with EU and UK law, NHS organisations have policies to support employees who wish to continue breastfeeding on return to work, which has numerous health benefits. The NHS employs many women of reproductive age, but anecdotally some face significant barriers in continuing breastfeeding. The objectives of the study were to describe awareness of relevant policies, describe breastfeeding behaviours and experience of staff in relation to these, and identify any areas for improvement. Fife NHS Board provides full hospital and primary care services in southeast Scotland and employs 8,000 staff.

Methods A questionnaire survey with quantitative and qualitative elements was sent from the Department of Human Resources to eligible employees of NHS Fife who had been on maternity leave between 2007-09. This was adapted from previous surveys with input from experts and was piloted in two stages. Those with serious complications e.g. stillbirth were excluded. Full ethical review was not required.

Results 651 women were eligible, with 87 questionnaires undelivered, and 342/564 (61\%) returned. Respondents were comparable to female employees of NHS Fife.; 203/329 (62\%) reported awareness of the breastfeeding and return to work policy. 26/342 (8\%) reported being offered information on support breastfeeding on return to work. $63 / 270(23 \%)$ who breastfed at all continued on their return and this was more common with increasing age, but not related to setting eg ward, community. 18/270 (7\%) would have breastfed longer if their employer had been more supportive, and 43/270 (16\%) cited returning to work as a reason they stopped.; 102/254 (40.2\%) felt not very or not at all confident to approach their employer regarding support. Women who were aware of the policies were significantly more likely to feel confident than women who were not, $(p<0.001) .4$ respondents used a suitable area to express milk and 3 made use of a designated fridge for storage.

Key themes described were that it was perceived that managers and other workers grudged the extra time for expressing milk or breastfeeding, and lack of facilities.

Conclusion Experience of staff was highly variable suggesting the policy was not implemented consistently. Suggested areas for improvement include: clearer information regarding policies with specific information on support for continued breastfeeding, line managers initiating discussion regarding the possibility of breastfeeding on return to work, and ensuring that women who do continue are not stigmatised by colleagues.

While these results may not be fully generalisable, it is likely that there are similar issues for NHS employees more widely.

\section{PS24 NUTRITIONAL STATUS IN RELATION TO CARIES EXPERIENCE AMONG PRIMARY SCHOOL IRAOI CHILDREN IN THE MIDDLE REGION OF IRAQ}

doi:10.1136/jech-2012-201753.123

'Ban S Diab, 'SK El Samarrai, 'WS Al-Alousi, ${ }^{2} \mathrm{ASD}$ Al-Radha. ${ }^{1}$ College of Dentistry, University of Baghdad, Baghdad, Iraq; ${ }^{2}$ College of Dentistry, Al- Mustansiriyah University, Baghdad, Iraq

Background Malnutrition when occurs during development period may cause irreversible effect on developing dental tissues leading to dental caries. The aims of the present study were to evaluate the nutritional status of primary school children and its influence on dental caries. 